\title{
Interactive comment on "A dynamical reconstruction of the Last Glacial Maximum ocean state constrained by global oxygen isotope data" by Charlotte Breitkreuz et al.
}

\section{Anonymous Referee \#4}

Received and published: 24 June 2019

In the study a combined model - data approach is used to get a better insight into the state of the ocean circulation during the Last Glacial Maximum (LGM). The authors use benthic and planktic oxygen isotope and SST data compilations and a general coupled ocean circulation model with a new data assimilation method that improves the match between proxy data and model results. The findings of the study corroborate new ideas that North Atlantic Deepwater (NADW) reached into the deep Atlantic during the LGM, rather than being replaced by its southern hemisphere counterpart (Antarctic Bottom Water). Furthermore, the study tests the sensitivity of the Atlantic Meridional Overturning Circulation (AMOC) on changes in different ocean regions and suggests that variability in the Atlantic and the Southern Ocean are more important for AMOC

Printer-friendly version

Discussion paper 
changes than the Pacific and Indian Ocean. Given the current controversial discussion of the deepwater distribution during the LGM, the findings of the study are of major interest to the paleoceanography community.

Despite the importance of the study, there are several points that need to be addressed before publishing:

1. The manuscript addresses a wide palaeoceanographic audience that increases the need of explaining the model set up and design in a comprehensive way. Currently, it is difficult to follow the methods section. The description of the datasets used for the assimilation are intermingled with model set up paragraphs. Thus, it does not become clear which dataset was used for what purpose. Furthermore, for the sensitivity experiments, it does not become clear which parameters were changed, in which way, and how the reported time steps were generated. I suggests to rewrite these parts of the method section. Additionally, it would be beneficial to a non- expert reader to add a flow chart that visualizes the different steps of the modeling approach.

2. It is difficult to follow the content of some of the figures, as information is missing and terms are not used consistently. Please make sure that the figures with captions are self- explanatory.

3. The manuscript discussion is heavily focused on the evaluation of the models performance rather than on the discussion of ocean circulation as proposed in the abstract. In order to achieve the latter, a more circulation focused discussion under consideration of additional references e.g. Oppo et al. 2018, Adkins, 2013 and suggestions of reviewer 1 is needed.

4. The adjoint AMOC sensitivity studies discussion needs to be better linked to the data and to existing studies.

5. Error estimates for temperature and $\mathrm{d} 180$ values are missing

6. Please make sure that the data for the data compilation is available to the reviewers.

Interactive

comment
Discussion paper

Printer-friendly version

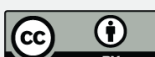


In addition to the more general comments, in the following you find line to line comments

P2 line 3-5 "Many studies indicate. . .(Lynch-Stieglitz et al., 2007)" please add additional studies

P2 line 5 please add Oppo et al., 2018

Interactive

comment

P2 line 14-16 It is difficult to follow this sentence as a non- modeler, please rephrase in order to address a wider audience.

P2 line 21-25 it is difficult to follow the paragraph, consider rephrasing

P3 line 7 exclusion of Mediterranean Sea, Please explicitly state that MOW inclusion resulted in insignificant changes of the results (Völpel et al. 2018), instead of referring vaguely to the literature. Although I guess, the model might show different results once the vertical resolution is increased and allows for a "proper" simulation of the MOW. Given the ongoing discussions on MOW importance for the AMOC recovery, it might be worth to address in the future.

P3 section 2.1 Model: Please specify the 15 vertical levels

P3 Line 17 to P4 line 4 It is difficult to follow this paragraph, rearranging the order of the paragraph starting with the general sentence on $\mathrm{d} 180$, before going into the details.

P4 line 2 "With the use of anomalies" anomalies between what? Please be more specific

P4 line 4-10 it is very difficult to follow this paragraph are you using the MARGO SST data to test SST or to calculate d180 from it?

P4 line 11-12 Maybe rephrase into "An overview of the used datasets can be found..." please provide citation on all datasets also for those used in Breitkreusz et al. 2019 under review (this might be done in an SI file) 
P4 line 13 "the same model grid" the same as used in this study?

P6 line 24-25 To me this detail belongs to chapter 2.2 Why are you using the ice volume correction of Duplessy (2002) instead of the new dataset of Austermann et al. (2013)

$P 7$ Please mention earlier how AMOC was calculated P 10 Figure 2: figure caption is not conclusive: what do the upper panels show? Is "First guess" are equal to "assimilated data"? If so please be consistent with the terms and describe the dataset from left to right. Please make sure that the figures are self- explanatory

P10 line 13 to p11 line 3 this paragraph belongs to methods P11 Figure 3 Please provide uncertainty of the data (either in table 1 or in figure caption 3 ).

P11 line $18 \mathrm{ff}$ Please define which the transact across Atlantic Ocean, in the text and add longitude and width of section. Figure 4 Please be careful using a $32.5^{\circ} \mathrm{W}$ transact through the Atlantic, it is neglecting the well- known differences between the eastern and western Atlantic basin, and might lead to a spatial bias. Although this difference might even not be resolved in the vertical resolution of the model, it needs to be addressed. As previously mentioned, it would be beneficial to add some information about the vertical layer distribution to enable the reader to evaluate the model performance on deepwater bathymetry and associated circulation changes.

$P 13$ line 1 to $p 15$ line 7 It is difficult to follow this paragraph. The set-up of the is unclear from the methods section which parameters were changed in order to perform the sensitivity experiments. Thus, it remains to the guess of the reader how the relation between potential T, salinity and AMOC was investigated. Furthermore, the temporal and spatial variability of SST and SSS is only partially mentioned (How do the absolute temperatures differ between the different runs?)

P14 lines 15-16 please provide references

$\mathrm{P} 15$ line 16 please provide numbers for $\mathrm{T}$ changes

Discussion paper

P18 line 16ff The discussion of different results model set ups in the end of the section

Interactive

comment

Printer-friendly version

C4 
leaves the reader with the message that the previously discussed deepwater distribution patterns might just be a matter of calculation and model set up. This may rise the question whether your results are trustworthy at all. In order to strengthen your arguments, you might want to place the discussion about mismatches with the work presented in Breitkreuz et al. 2019 (in review) and other modeling studies to the beginning of the section and emphasize the advantages of the adjoint method presented here.

P19 line 16 Why seems the Atlantic Ocean to be more important? Here a link to your model results is missing.

P 19 line 20 To me it remains unclear how you can evaluate sensitivities for a time span of 150 years, if your cost function only covers 60 years.

Please control whether terms are used consistently.

\section{References}

Austermann, J., J. X. Mitrovica, K. Latychev, and G. A. Milne (2013), Barbados-based estimate of ice volume at Last Glacial Maximum affected by subducted plate, Nature Geosci, 6(7), 553-557, 10.1038/ngeo1859 Oppo, D. W., G. Gebbie, K.-F. Huang, W. B. Curry, T. M. Marchitto, and K. R. Pietro (2018), Data Constraints on Glacial Atlantic Water Mass Geometry and Properties, Paleoceanography and Paleoclimatology, 33(9), 1013-1034, 10.1029/2018PA003408. Adkins, J. F. (2013), The role of deep ocean circulation in setting glacial climates, Paleoceanography, 28(3), 539-561, 10.1002/palo.20046.

Interactive comment on Clim. Past Discuss., https://doi.org/10.5194/cp-2019-52, 2019. 